Supplement of Hydrol. Earth Syst. Sci., 23, 4869-4889, 2019

https://doi.org/10.5194/hess-23-4869-2019-supplement

(C) Author(s) 2019. This work is distributed under

the Creative Commons Attribution 4.0 License.

(c) (1)

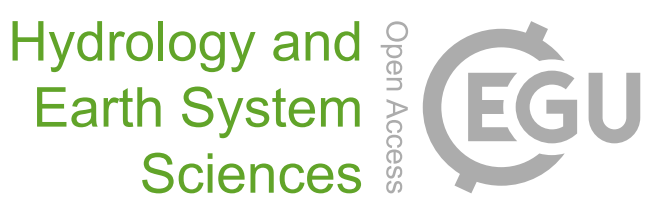

Supplement of

\title{
Spatio-temporal relevance and controls of preferential flow at the landscape scale
}

Dominic Demand et al.

Correspondence to: Dominic Demand (dominic.demand@hydrology.uni-freiburg.de)

The copyright of individual parts of the supplement might differ from the CC BY 4.0 License. 
Table S1: Additional textural information and standard deviation (sd) of the soils sampled in the different landscape units.

\begin{tabular}{|c|c|c|c|c|c|c|c|c|}
\hline \multirow{3}{*}{ Slate } & & Sand & sd & Silt & sd & Clay & sd & No. of samples \\
\hline & Forest & 10 & 15 & 52 & 10 & 38 & 6 & 38 \\
\hline & Grassland & 11 & 13 & 49 & 9 & 40 & 6 & 17 \\
\hline \multirow{4}{*}{ Marl } & Forest $<30 \mathrm{~cm}$ & 32 & 25 & 45 & 21 & 23 & 8 & 16 \\
\hline & Forest $>30 \mathrm{~cm}$ & \multicolumn{6}{|c|}{ estimated by field test $>50 \%$ Clay } & 0 \\
\hline & Grassland $<30 \mathrm{~cm}$ & 24 & 16 & 46 & 14 & 30 & 6 & 46 \\
\hline & Grassland $>30 \mathrm{~cm}$ & 16 & 12 & 36 & 7 & 48 & 16 & 12 \\
\hline \multirow{2}{*}{ Sandstone } & Forest & 64 & 11 & 20 & 8 & 16 & 5 & 32 \\
\hline & Grassland & 58 & 5 & 23 & 4 & 19 & 2 & 8 \\
\hline
\end{tabular}


Table S2: van Genuchten (1980) parameters optimized by Sprenger et al. (2016). Site IDs with an "x" indicate sites were the best fit of the respective geology was used, because the site was not optimized. Subscripts indicate the respective depth of the parameter. $K_{s}$ is given in cm day ${ }^{-1}$ and $\alpha$ in $\mathrm{cm}^{-1}$.

\begin{tabular}{|c|c|c|c|c|c|c|c|c|c|c|}
\hline Site ID & $\theta_{\mathrm{s} 10}$ & $\alpha_{10}$ & $\mathbf{n}_{10}$ & $\mathrm{~K}_{\mathrm{s} 10}$ & $\theta_{\mathrm{s} 30}$ & $\alpha_{30+50}$ & $\mathbf{n}_{30+50}$ & $\mathrm{~K}_{\mathrm{s} 30}$ & $\boldsymbol{\theta}_{\mathrm{s} 50}$ & $\mathrm{~K}_{\mathrm{s} 50}$ \\
\hline M_A & 0.530 & 0.010 & 1.309 & 842 & 0.436 & 0.011 & 1.311 & 845 & 0.496 & 185 \\
\hline M_B & 0.598 & 0.040 & 1.213 & 10 & 0.409 & 0.015 & 1.075 & 979 & 0.469 & 765 \\
\hline M_C_x & 0.545 & 0.027 & 1.265 & 314 & 0.447 & 0.029 & 1.200 & 675 & 0.442 & 293 \\
\hline M_D_x & 0.545 & 0.027 & 1.265 & 314 & 0.447 & 0.029 & 1.200 & 675 & 0.442 & 293 \\
\hline M_E & 0.580 & 0.070 & 1.190 & 423 & 0.499 & 0.077 & 1.178 & 417 & 0.456 & 49 \\
\hline M_F_x & 0.545 & 0.027 & 1.265 & 314 & 0.447 & 0.029 & 1.200 & 675 & 0.442 & 293 \\
\hline M_G_x & 0.545 & 0.027 & 1.265 & 314 & 0.447 & 0.029 & 1.200 & 675 & 0.442 & 293 \\
\hline M_H & 0.453 & 0.020 & 1.193 & 451 & 0.452 & 0.074 & 1.185 & 985 & 0.439 & 15 \\
\hline M_I & 0.544 & 0.029 & 1.236 & 356 & 0.390 & 0.012 & 1.154 & 360 & 0.374 & 436 \\
\hline M_J & 0.600 & 0.013 & 1.237 & 82 & 0.491 & 0.011 & 1.176 & 700 & 0.419 & 588 \\
\hline M_K & 0.512 & 0.004 & 1.476 & 35 & 0.451 & 0.003 & 1.321 & 438 & 0.440 & 10 \\
\hline S_A & 0.547 & 0.008 & 1.316 & 180 & 0.326 & 0.004 & 1.299 & 348 & 0.434 & 715 \\
\hline S_B & 0.503 & 0.071 & 1.265 & 595 & 0.353 & 0.017 & 1.287 & 643 & 0.358 & 550 \\
\hline S_C & 0.556 & 0.087 & 1.249 & 53 & 0.338 & 0.015 & 1.195 & 689 & 0.321 & 172 \\
\hline S_D & 0.538 & 0.039 & 1.328 & 10 & 0.400 & 0.028 & 1.319 & 915 & 0.480 & 130 \\
\hline S_E & 0.562 & 0.036 & 1.379 & 375 & 0.400 & 0.012 & 1.383 & 418 & 0.339 & 24 \\
\hline S_F_X & 0.526 & 0.038 & 1.335 & 531 & 0.377 & 0.011 & 1.384 & 648 & 0.432 & 360 \\
\hline S_G & 0.577 & 0.100 & 1.278 & 769 & 0.302 & 0.023 & 1.285 & 1000 & 0.437 & 373 \\
\hline S_H & 0.520 & 0.071 & 1.296 & 646 & 0.419 & 0.006 & 1.584 & 853 & 0.495 & 233 \\
\hline S_I & 0.569 & 0.003 & 1.526 & 580 & 0.409 & 0.010 & 1.221 & 1000 & 0.448 & 383 \\
\hline S_J_X & 0.526 & 0.038 & 1.335 & 531 & 0.377 & 0.011 & 1.384 & 648 & 0.432 & 360 \\
\hline S_K_x & 0.526 & 0.038 & 1.335 & 531 & 0.377 & 0.011 & 1.384 & 648 & 0.432 & 360 \\
\hline S_L_X & 0.526 & 0.038 & 1.335 & 531 & 0.377 & 0.011 & 1.384 & 648 & 0.432 & 360 \\
\hline S_M & 0.516 & 0.015 & 1.298 & 525 & 0.440 & 0.002 & 1.894 & 249 & 0.436 & 736 \\
\hline S_O & 0.524 & 0.016 & 1.325 & 748 & 0.412 & 0.010 & 1.259 & 612 & 0.500 & 144 \\
\hline S_P & 0.427 & 0.013 & 1.267 & 351 & 0.413 & 0.002 & 1.895 & 244 & 0.437 & 89 \\
\hline S_Q & 0.543 & 0.021 & 1.314 & 278 & 0.478 & 0.010 & 1.382 & 501 & 0.479 & 469 \\
\hline S_R_x & 0.526 & 0.038 & 1.335 & 531 & 0.377 & 0.011 & 1.384 & 648 & 0.432 & 360 \\
\hline S_S & 0.477 & 0.048 & 1.270 & 931 & 0.300 & 0.016 & 1.229 & 872 & 0.409 & 150 \\
\hline S_T & 0.470 & 0.012 & 1.374 & 1000 & 0.314 & 0.006 & 1.335 & 728 & 0.469 & 426 \\
\hline S_U & 0.559 & 0.003 & 1.557 & 513 & 0.433 & 0.002 & 1.365 & 757 & 0.411 & 999 \\
\hline S_V_X & 0.526 & 0.038 & 1.335 & 531 & 0.377 & 0.011 & 1.384 & 648 & 0.432 & 360 \\
\hline S_W & 0.522 & 0.060 & 1.319 & 948 & 0.300 & 0.017 & 1.219 & 545 & 0.454 & 164 \\
\hline Sa_A & 0.516 & 0.038 & 1.340 & 494 & 0.450 & 0.006 & 1.686 & 795 & 0.500 & 1000 \\
\hline Sa_B & 0.469 & 0.043 & 1.187 & 439 & 0.417 & 0.017 & 1.170 & 848 & 0.373 & 627 \\
\hline Sa_C & 0.596 & 0.075 & 1.235 & 585 & 0.382 & 0.003 & 1.425 & 617 & 0.445 & 931 \\
\hline Sa_D & 0.456 & 0.030 & 1.298 & 632 & 0.404 & 0.009 & 1.325 & 812 & 0.496 & 1000 \\
\hline Sa_E & 0.521 & 0.050 & 1.204 & 431 & 0.366 & 0.003 & 1.205 & 98 & 0.332 & 318 \\
\hline Sa_F & 0.573 & 0.001 & 1.818 & 10 & 0.469 & 0.001 & 1.650 & 72 & 0.492 & 995 \\
\hline Sa_G & 0.546 & 0.033 & 1.228 & 528 & 0.319 & 0.005 & 1.194 & 132 & 0.470 & 532 \\
\hline $\mathrm{Sa} \_\mathrm{H}$ & 0.464 & 0.061 & 1.225 & 234 & 0.338 & 0.013 & 1.384 & 444 & 0.411 & 924 \\
\hline Sa_I & 0.529 & 0.038 & 1.329 & 598 & 0.400 & 0.005 & 1.354 & 501 & 0.400 & 877 \\
\hline Sa_J & 0.452 & 0.079 & 1.165 & 268 & 0.400 & 0.003 & 1.231 & 456 & 0.464 & 977 \\
\hline Sa_K & 0.493 & 0.022 & 1.286 & 181 & 0.425 & 0.020 & 1.297 & 805 & 0.495 & 718 \\
\hline Sa_L & 0.456 & 0.054 & 1.242 & 915 & 0.427 & 0.028 & 1.347 & 509 & 0.486 & 477 \\
\hline
\end{tabular}


Table S3: Number of infiltration events for the different response classifications and depth. These infiltration events are the data basis for Table 3 and 4 and Figure 4, 8, 9. Note: a SR 50 response results always in two measured maximum pore water velocity $\left(v_{\max }\right), 10-30$ and $30-50 \mathrm{~cm}$.

\begin{tabular}{ccccccc|c}
\hline & \multicolumn{2}{c}{ Slate } & \multicolumn{2}{c}{ Marl } & \multicolumn{2}{c}{ Sandstone } & \\
& Forest & Grassland & Forest & Grassland & Forest & Grassland & Total \\
\hline SR 30 & 319 & 113 & 68 & 59 & 198 & 64 & 821 \\
SR 50 & 326 & 92 & 84 & 57 & 262 & 89 & 910 \\
NSR 10 - 50 & 103 & 11 & 34 & 12 & 42 & 13 & 215 \\
NSR 30 & 174 & 48 & 60 & 43 & 39 & 17 & 381 \\
NSR 50 & 118 & 16 & 24 & 4 & 18 & 7 & 187 \\
NC - no resp. & 1285 & 529 & 265 & 480 & 730 & 362 & 3651 \\
NC- 10 cm & 650 & 312 & 198 & 197 & 582 & 146 & 2085 \\
\hline Total & 2975 & 1121 & 733 & 852 & 1871 & 698 & 8250 \\
\hline
\end{tabular}

Table S4: Number of infiltration events for the four defined volumetric soil moisture quartiles. These infiltration events are the data basis for Figure 6.

\begin{tabular}{cccccc}
\hline & & & \multicolumn{3}{c}{ quartile of vol. soil moisture } \\
& & $\mathbf{1}$ - lowest & $\mathbf{2}$ & $\mathbf{3}$ & $\mathbf{4}$ - highest \\
\hline \multirow{2}{*}{ Slate } & Forest & 769 & 792 & 835 & 579 \\
& Grassland & 290 & 285 & 306 & 240 \\
\hline \multirow{2}{*}{ Marl } & Forest & 178 & 192 & 182 & 181 \\
& Grassland & 211 & 232 & 224 & 185 \\
\hline \multirow{2}{*}{ Sandstone } & Forest & 563 & 467 & 486 & 355 \\
& Grassland & 156 & 171 & 210 & 161 \\
\hline
\end{tabular}


Table S5: Number of infiltration events recorded in total in each month and the minimum and maximum number recorded in single years. These infiltration events are the data basis for Figure 7.

\begin{tabular}{|c|c|c|c|c|c|c|}
\hline & \multicolumn{3}{|c|}{ Forest } & \multicolumn{3}{|c|}{ Grassland } \\
\hline & total & min. single year & max. single year & total & min. single year & max. single year \\
\hline Jan & 487 & 22 & 167 & 176 & 17 & 82 \\
\hline Feb & 408 & 25 & 166 & 142 & 8 & 63 \\
\hline Mar & 266 & 6 & 97 & 144 & 2 & 77 \\
\hline Apr & 389 & 24 & 149 & 194 & 15 & 70 \\
\hline May & 490 & 60 & 141 & 336 & 12 & 140 \\
\hline Jun & 482 & 66 & 115 & 240 & 39 & 62 \\
\hline Jul & 425 & 50 & 133 & 210 & 29 & 63 \\
\hline Aug & 641 & 70 & 202 & 268 & 21 & 107 \\
\hline Sep & 375 & 52 & 107 & 133 & 5 & 55 \\
\hline Oct & 523 & 59 & 185 & 250 & 12 & 90 \\
\hline Nov & 515 & 68 & 145 & 280 & 31 & 100 \\
\hline Dec & 578 & 35 & 249 & 298 & 18 & 129 \\
\hline
\end{tabular}

Table S6: Number of maximum pore water velocity records for the four defined volumetric soil moisture quartiles used for Fig. 11. The number includes $v_{\max }$ observed at both depth $(10-30$ and $30-50 \mathrm{~cm})$.

\begin{tabular}{cccccc}
\hline & & & \multicolumn{3}{c}{ quartile of vol. soil moisture } \\
& & $\mathbf{1}$ - lowest & $\mathbf{2}$ & $\mathbf{3}$ & $\mathbf{4}$ - highest \\
\hline \multirow{2}{*}{ Slate } & Forest & 168 & 299 & 302 & 202 \\
& Grassland & 58 & 67 & 99 & 73 \\
\hline \multirow{2}{*}{ Marl } & Forest & 47 & 77 & 61 & 51 \\
& Grassland & 41 & 87 & 37 & 8 \\
\hline \multirow{2}{*}{ Sandstone } & Forest & 210 & 172 & 177 & 163 \\
& Grassland & 27 & 62 & 83 & 70 \\
\hline
\end{tabular}


Slate

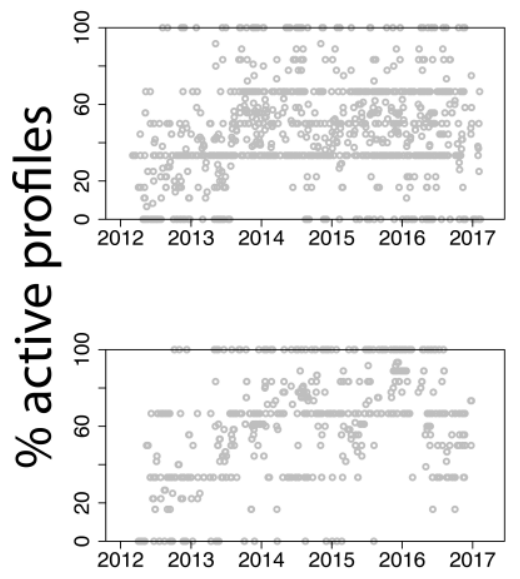

Marl
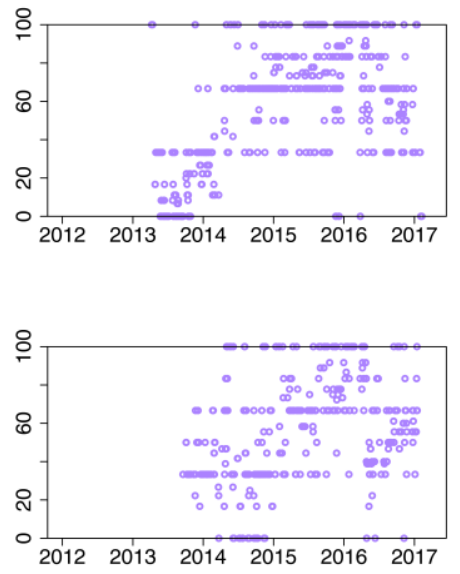

Sandstone
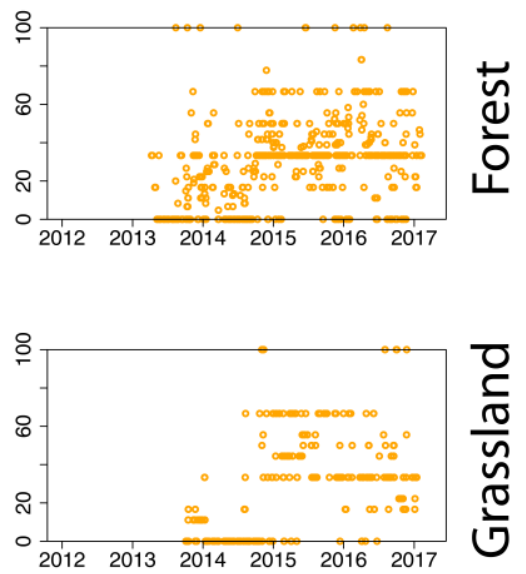

Figure S1: Proportion of soil moisture profiles which met all quality criteria (active) for profiles and days with registered rainfall (= \# active profiles / \#profile that registered rainfall). 